\title{
EFFICIENT SPEED GOVERNOR FOR BLOWER MOTOR
}

\author{
Nagaraj Hediyal ${ }^{1}$, Sujatha Nagaraj ${ }^{2}$ \\ ${ }^{I}$ Chief Executive Officer, eNLiven Technologies, Bangalore-32., Karnataka, India \\ ${ }^{2}$ Senior Lecturer, Dept of Electronics and Communication, M N Technical Institute, KG Halli, Bangalore-15
}

\begin{abstract}
This paper presents an efficient speed governor for the blower motor used in the air conditioning of electric vehicles (EV). Performance optimization of blowers offers tremendous potential for energy saving and hence the running cost. Pulse width modulation (PWM) technique is one of the most economical and widely used techniques in the motor control applications. SG 3525 device is used to generate the required PWM signals for the regulation of the blower motor speed. The PWM signal with 0-100\% duty ratio control governs the semiconductor switches such as MOSFETs in controlling the supply voltage to the motor. The proposed control scheme governs the speed by varying the reference voltage to the $S G 3525$ and also protects the motor from high current as the scheme monitors the current flow through the motor indirectly via gate control signal of the semiconductor switches.
\end{abstract}

Keywords: Pulse width modulation (PWM), Blower, Electric Vehicle (EV), SG 3525, MOSFET, Duty ratio.

\section{INTRODUCTION}

Nowadays, "GO GREEN" has become the universal chant of everybody to protect the environment from all sorts of pollution. The major contributors for the environmental degradation are vehicles, industries, mismanagement of wastages etc. There is a tremendous amount of research work under taken to find the solution to pollution control.

In order to protect the environment electric vehicles (Battery operated) are being considered along with the other type of existing vehicles. Most of the vehicles are provided with air conditioning systems for the human comfort. These systems and other electrical accessories such as lighting system, wind screen system, automatic windows system play a vital role in the maintenance and running cost of the vehicle. Due to the over demand and market competition the vehicle manufacturers are looking for cost effective solutions. These electrical sub systems must be compact, reliable, long lasting and technologically advanced. When it comes to the technologically advanced system, designs become complex and costlier. The technology has evolved over the years and largely the research is focussed on improving the performance of the electric vehicles which in turn takes care of the pollution control.

In this paper to improve the performance of the blower motor in air conditioning system, PWM based speed regulator is devised. The SG 3525 is a single chip solution to have a PWM control signals and also brings simplicity in the design.

\section{RELATED WORK}

Green Refrigeration and Air Conditioning Systems are gaining potential in this regard [1]. As brought out in [1] major industries can implement the green refrigeration and air conditioning systems towards the pollution control from their end. It is clearly explained in [1] how the thermoelectric refrigeration could be adapted in a cost effective manner compared to other thermoelectric refrigeration methods.

Similar efforts needs to be followed by the vehicle manufacturers to control the pollution as the number of vehicles on road are uncontrollably increasing. Due to this vehicle manufacturers are focussing on the electric vehicles which contribute very less percentage of pollution to the environment. The various kinds of electric sub systems used in the manufacturing of EVs are clearly studied and focus is on reliable, technologically advanced comfort, cost effective EVs [2]. It has been carefully presented how the A/C system performance contributes to the EV in terms of technology, cost etc. The importance of the blower evaporator in achieving the performance of $\mathrm{A} / \mathrm{C}$ system is also well presented [2]. As a matter of fact the blower speed control in air conditioning of electric vehicle plays an important role in providing the comfort to the human being.

The importance and the advantages of PWM converters in various applications such as inverter, speed control etc have been well dealt in $[3,4,5,6]$. The use of FPGA, CPLD, [3, 4] can only help in reducing the cost in industrial applications with PWM techniques but not in EVs. In case of EVs it is desired to have low cost, less complex but advanced controllers and not compromising the human comfort. This paper presents the "Efficient Speed Governor for Blower Motor" used in A/C system of EVs and other type of vehicles. 


\section{METHODS OF SPEED CONTROLS}

Cooling and dehumidification are the two main functions done by the air conditioning system. Adjusting the blower speed during air conditioner operation can offer many advantages in terms of energy consumption, comfort, humidity control etc. There are many speed control methods available from the implementation point of view. These are:

1. Variable speed control,

2. Passive device control,

3. Solid State control and

4. Mechanical device control.

The blower speed can be simply switched either manually or electronically. In most of the A/C systems, blower motor has three speeds namely, high, medium and low. The general mechanism used in the speed control is shown in figure 1 below.

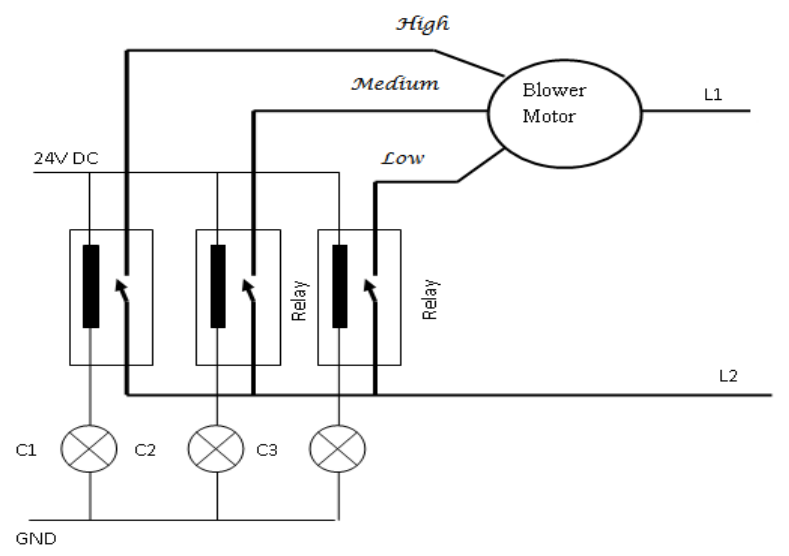

Fig-1 Speed Control Mechanism

The mechanism shown in figure-1 is little complex and has many devices to handle. $\mathrm{C} 1, \mathrm{C} 2$ and $\mathrm{C} 3$ are the controls to be operated either manually or electronically. These types of systems are in use since long, however presently many of the methods have been combined to obtain sustainable controls. Operating the blower motor in high, medium or low speed mode has advantages in terms of running cost and also comfort. Above method seems to be little cumbersome and may cause noise, discomfort, because less removal of moisture. Many of the above controls have been combined to implement speed control strategy. This necessitates the auto mode of operation with a smooth and comfortable mechanism to handle the operation of the blower motor in $\mathrm{A} / \mathrm{C}$ system.

\section{PROPOSED METHOD}

The proposed method uses a variable speed control through a PWM based technology where duty ratio controls the applied voltage to the blower motor and hence the speed. System makes it very simple and compact solution. The block diagram of the proposed system is shown in figure- 2 below.

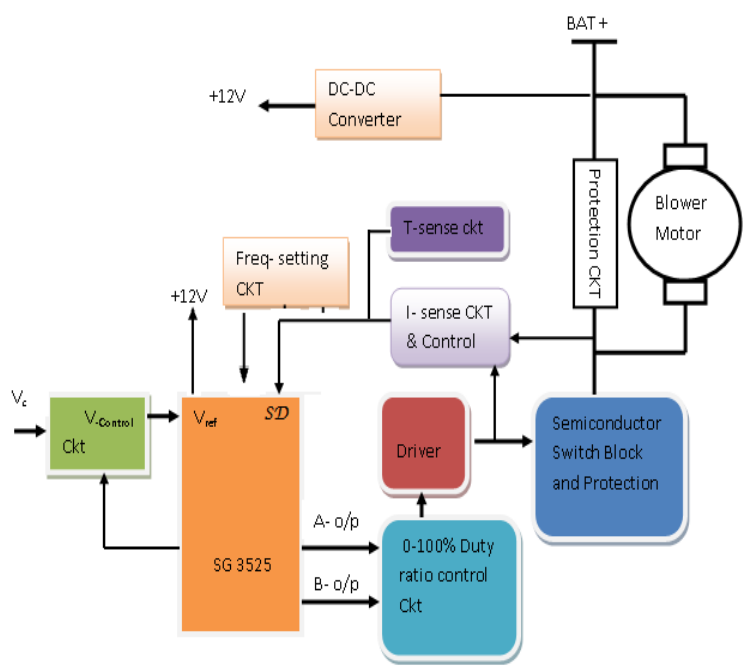

Fig-2 Proposed Block Diagram

The proposed system consists of DC-DC converter, current sense block, switch block, duty ratio control block, voltage reference block, driver block, frequency setting block, and temperature sense block.

\section{EXPERIMENTAL SETUP}

The experimental setup is shown in figure- $3 a$ and figure- $3 b$ below.

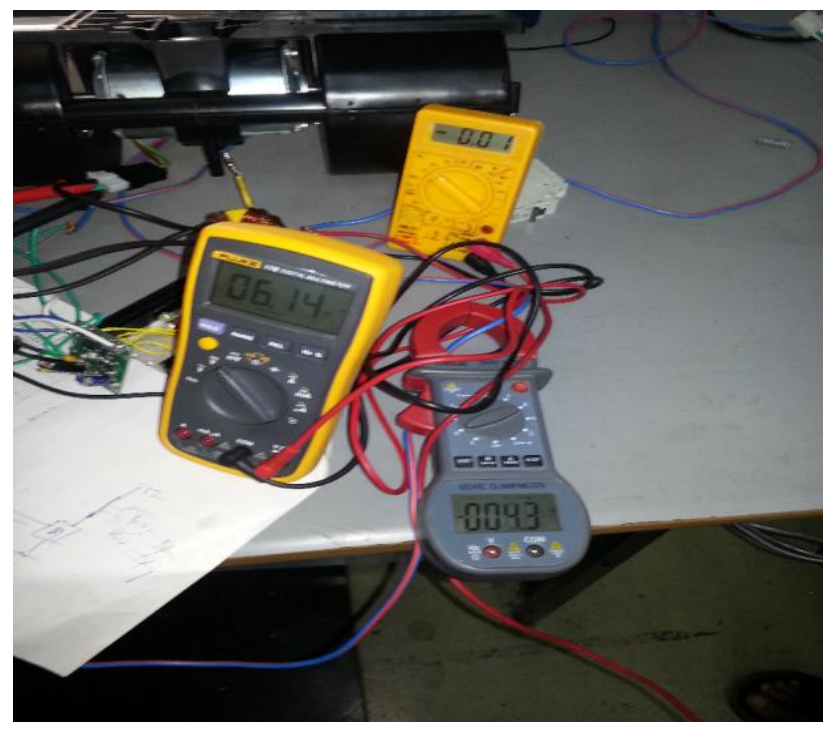

Fig-3a Prototype 


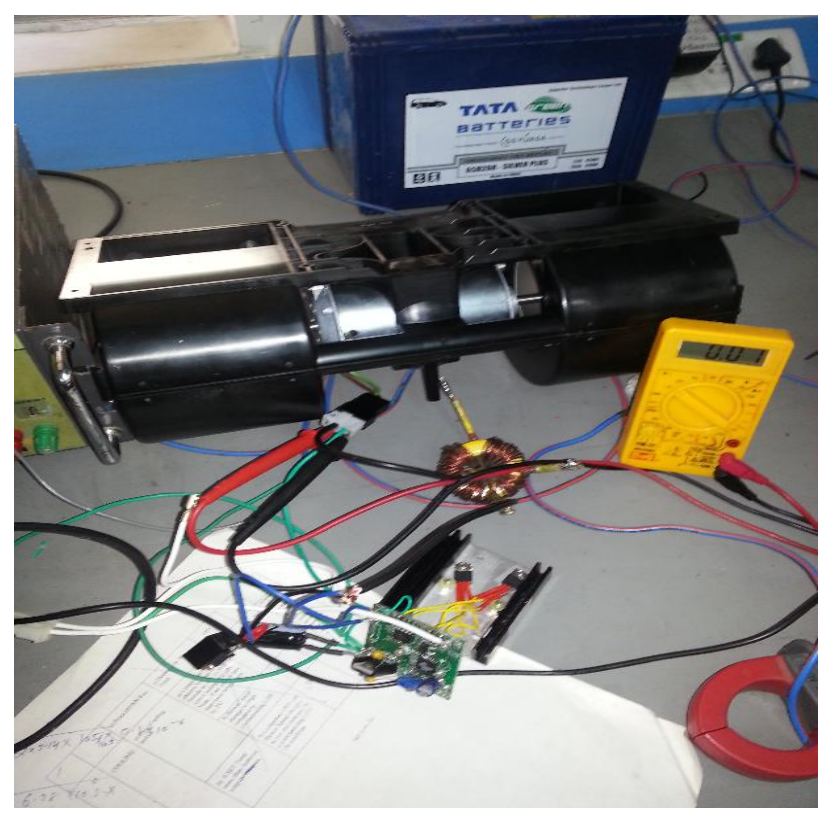

Fig-3b Prototype

The proposed prototype is $40 \mathrm{~mm} X 55 \mathrm{~mm}$ in size as shown in figure- $3 \mathrm{a}$ and $3 \mathrm{~b}$ above. The switching frequency is $33 \mathrm{KHz}$ and is more appropriate for this application. Generally, motor control switching frequency should be above $20 \mathrm{KHz}$ nominal. SG 3525 device is chosen as it makes the circuit to be more simple, robust and reliable due to reduced device count in the design of controller. There is current block which continuously senses the flow of current through the blower motor. This is done by the current sense through the gate drive signals used to switch the semiconductor switches such as MOSFET. There is a set of switches to share the current and both operate simultaneously. The duty ratio can be adjusted by varying the control voltage fed to the error amplifier input of the SG 3525 device. The control voltage $\left(\mathrm{V}_{\mathrm{c}}\right)$ can be fed externally to have a control over the duty ratio and hence the speed of the blower motor. The proposed system also provides the soft start and blower motor steadily attains the speed based on duty ratio.

Based on the duty ratio the voltage $\left(\mathrm{V}_{\mathrm{m}}\right)$ across the blower motor terminals varies and hence the current $\left(\mathrm{I}_{\mathrm{m}}\right)$. When the current is maximum the current sense block generates an error current signal adjusts the duty ratio automatically to reduce the voltage across the blower motor terminals and shuts down if it exceeds the over current limit. When the temperature of the heat sink exceeds $90^{\circ} \mathrm{C}$, system shuts down automatically. Thus the speed governor protects the blower motor from damage. Snubber is provided across the terminals of the blower motor to avoid the effect of loop current when the motor is in off condition. It has a control voltage input to feed the voltage from any other external source. This option is mandatory and to be applied or selected by the driver through a microcontroller based controllers.

\section{RESULTS AND DISCUSSION}

The main device used in the design of this governor is SG3525 with other circuitries. The output wave forms of SG3525 OUT-A and OUT-B are shown in figure-4 below.

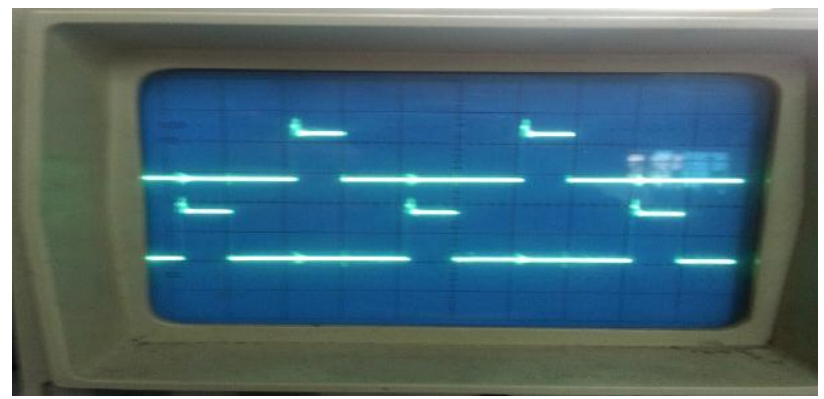

Fig-4 SG3525 PWM Output Wave forms

The PWM signal with 50\% and $95 \%$ duty cycle are shown in figure- 5 and figure- 6 respectively.

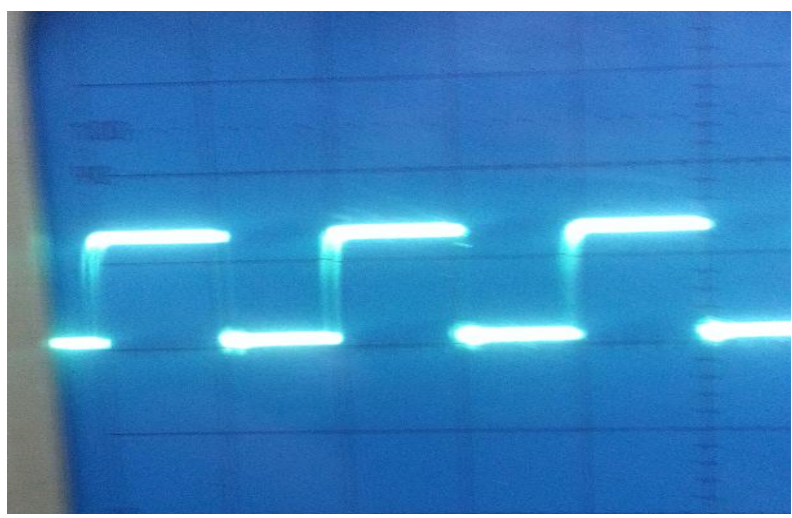

Fig-5, PWM Signal with 50\% Duty Ratio.

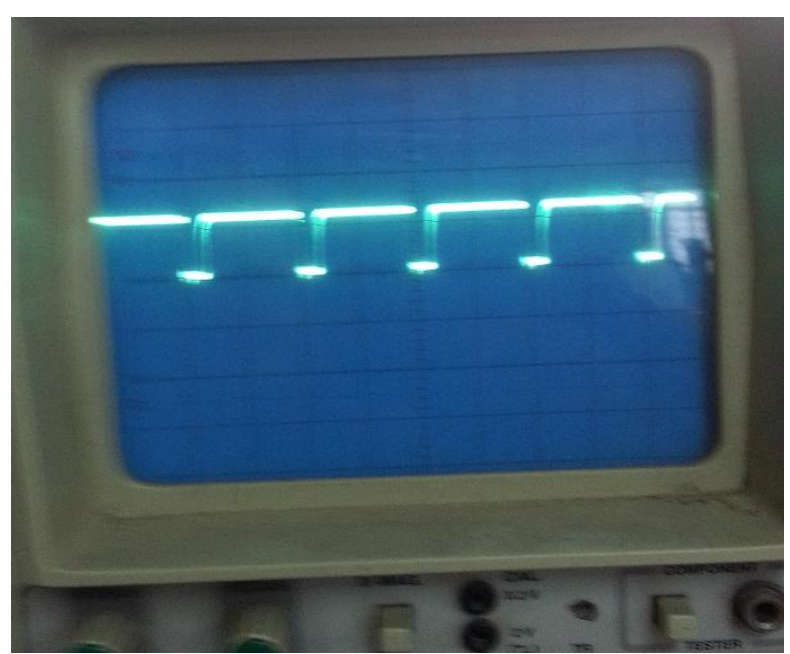

Fig-6, PWM Signal with 95\% Duty Ratio. 
The variation of control voltage and blower motor voltage is plotted and shown in figure-7 below.

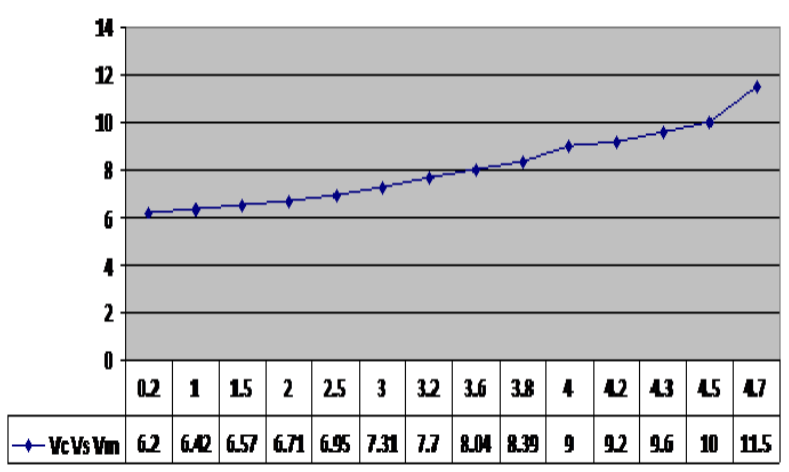

Fig-7 control Voltage Vs Blower Motor Voltage

The variation of control voltage with Blower Motor Current is shown in figure- 8 below.

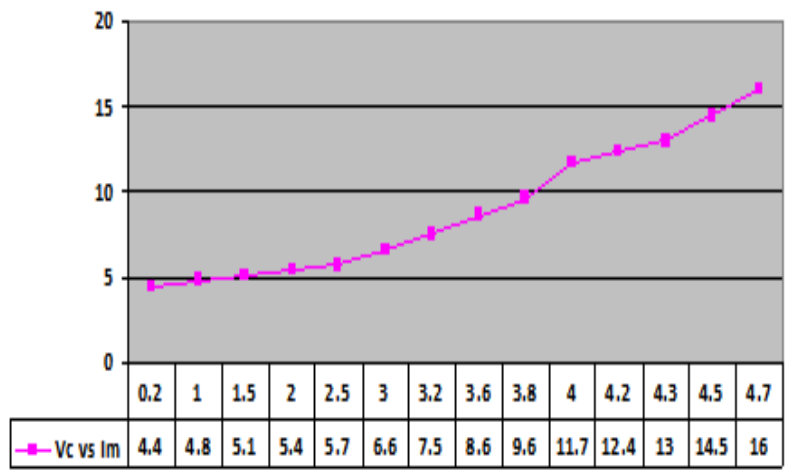

Fig-8 Control Voltage Vs Blower Motor Current

The relation of voltage and current of blower motor is shown in figure-9 below

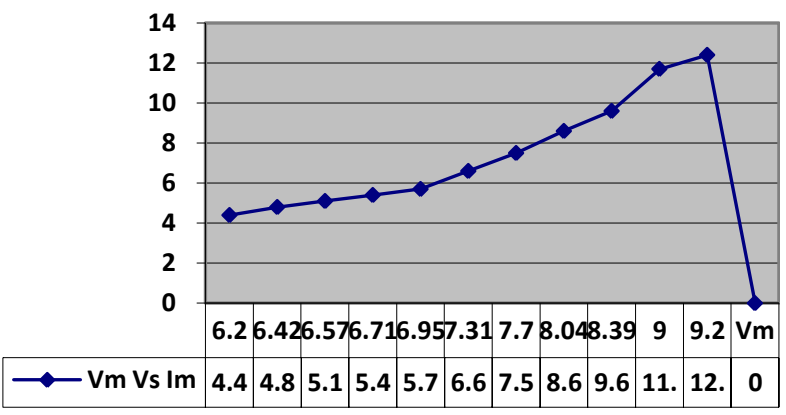

Fig-9 Relation between voltage and current of blower motor
The operating frequency of this controller is about $33 \mathrm{KHz}$ and the duty ratio is adjustable from $0-100 \%$. Speed regulator shuts down when the temperature of the heat sink is above $90 \mathrm{oC}$ and also when the current exceeds $16 \mathrm{~A}$ through the motor. The design is simple but efficient in achieving the required functions and no compromise on the price point of view.

\section{CONCLUSIONS}

The proposed "Efficient Speed Governor for Blower Motor" is completely justified in terms of its:

1. Low cost,

2. Less complex and compact,

3. Reliable,

4. Soft start.

5. Smooth speed regulation from low to high speed,

6. Thermal and over current protection.

\section{REFERENCES}

[1]. Monoj Kumar Rawat et al "A Review on Developments of Thermoelectric Refrigeration and Air Conditioning Systems: A Novel Potential Green Refrigeration and Air Condition Technology" Volume 3, Special Issue 3: ICERTSD 2013, Feb 2013, pages 362-367

[2]/ Po-Hsu Lin "Performance evaluation and analysis of EV Air-Conditioning System" World Electric Vehicle Journal (WEVJ) Vol.4- ISSN 2032-6653- (C) 2010 WEVA Page 000197.

[3]. Eftichios Koutroulis et al. "High-frequency pulse width modulation implementation using FPGA and CPLD ICs" Journal of Systems Architecture (JSA) 52 (2006) 332-344.

[4]. Jaroslav Dudrik et al. "High-Frequency Soft Switching DC-DC Converters for Voltage and Current DC Power Sources", Acta Polytechnica Hungarica Vol4, No.2, 2007 Page- 29-46

[5]. Kariappa B S et al. "FPGA Based Speed Control of AC Servomotor Using Sinusoidal PWM", International Journal of Computer Science and Network Secuirty (IJCSNS), Vol 8 No.10, October 2008 Page 346-350

[6]. Omokere et al. "Evaluating the Performance of a Single Phase PWM Inverter Using 3525A PWM IC", International Journal of Engineering Research \& Technology (IJERT), Vol. 1 Issue 4, June-2012.

\section{BIOGRAPHIES:}

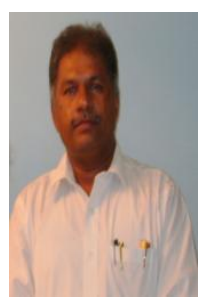

Nagaraj Hediyal received his BE (Electrical Engineering) (UVCE), and M.Tech (Computer Applications in Industrial Drives) (MSRIT) from Bangalore University and Visvesvaraya Technological University respectively. Presently he has assumed the role of Chief Executive Officer of eNLiven Technologies. He served as Vice PresidentTechnical, leading R \& D and Production divisions at Systems 
Aids Bangalore-43. Formerly, Technical Manager, CG-CoreEl Programmable Solutions Pvt Ltd, Bangalore. He served as Head of the Section (Encryption) \& Scientist, Core R \& D, ITI Ltd, Bangalore-16. He was the main \& leading designer of most of the encryption systems at ITI Ltd. The advanced high speed STM1, STM4and E3 ATM encryption systems were his brain child's to name a few. He has authored and co-authored number of proprietary encryption algorithms for Indian Defence networks used by Army, Navy and Air-force. He was honoured with letter of appreciations from both ITI Ltd and Indian Army. He is a fellow of IETE. His research interests are Cryptography, VLSI, Industrial Electronics and Embedded systems.

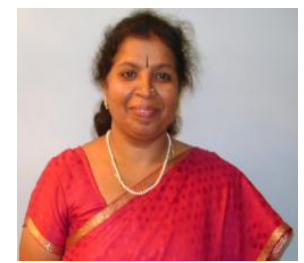

Mrs Sujatha Nagaraj received her BE (Instrumentation Technology) (Dr.AIT), from Bangalore University. At present she is serving as Sr. Lecturer, Dept of Electronics and Communication in $\mathrm{M} \mathrm{N}$ Technical Institute, Kammagondanahalli, Bangalore-15. Her research interests are Electronics Instrumentation, Industrial Automation, Biomedical Electronics, Counselling. 